\title{
The need for a new toxicity testing and risk analysis paradigm to implement REACH or any other large scale testing initiative
}

\author{
Bas J. Blaauboer • Melvin E. Andersen
}

Received: 16 November 2006 / Accepted: 19 December 2006 / Published online: 30 January 2007

(C) Springer-Verlag 2007

In a paper last year in Archives of Toxicology, "Toxicological comments to the discussion about REACH", a group of distinguished European community toxicologists (Greim et al. 2006) expressed their opinions on discussions about the extent to which in vitro studies and considerations of structure activity relationships provide sufficient information to waive repeated exposure studies in animals. The authors argued that critical data needed for hazard identification and risk assessment can only be developed by conducting repeat dose studies in animals. They consider the data sets from these studies to be essential for risk assessment. In their abstract, they note that, "The evidence that available alternatives would support such replacement is weak. Progress to improve their value for risk assessment purposes is bound to be slow because the issues are very complex. As a group of European toxicologist we strongly support the need for more research support in these areas, but we believe that over claims for progress is damaging their development."

We appreciate the concerns raised about too rapid replacement of these repeat studies without a clear path for how data sets from in vitro or SAR evaluations

B. J. Blaauboer $(\square)$

Institute for Risk Assessment Sciences (IRAS),

Utrecht University, PO Box 80.176, 3508 TD Utrecht,

The Netherlands

e-mail: b.blaauboer@iras.uu.nl

M. E. Andersen ( $\square)$

The Hamner Institutes for Health Sciences, PO Box 12137,

Research Triangle Park, NC, 27709-2137, USA

e-mail: MAndersen@thehamner.org would supplant these more traditional toxicity studies. However, we disagree strongly with the conclusions of the commentary on the state of development of these methods for decision making. More importantly, we feel compelled to take exception with several points presented or inferences made in this paper. The points that require some discussion are: (1) current testing/risk assessment strategies represent an optimal approach for making decisions regarding human health risks of chemicals; (2) the goal of a replacement is to develop tests that provide perfect correspondence with the current in vivo toxicity testing approaches; (3) in vitro methods cannot provide information regarding doseresponse necessary for risk assessments; and (4) approaches to introduce new methods for toxicity testing and risk assessment reflect consideration of cost reduction and decreased animal use rather than decisions taken on the basis of toxicological considerations. We briefly discuss each point below.

\section{Current testing/risk assessment strategies represent an optimal approach for making decisions regarding human health risks of chemicals}

The current testing strategies, relying almost exclusively on results from animal studies, are an amalgam of testing requirements developed piecemeal starting in the mid-1900s. These studies utilize the highest tolerated doses in multiple animal species and develop dose-response curves to assess no observed effect (NOEL) or no observed adverse effect levels (NOAEL) for a variety of physiological endpoints. These approaches may be widely accepted, but they are not at all validated for the purposes of predicting 
human health risks. Together with safety factors, these NOAELs and NOELs may provide reassurance to many that there will be little risks in exposed humans. However, it is simply wrong to consider animal toxicity tests as some gold standard against which all in vitro or in silico methods will be judged.

\section{The goal of a replacement is to develop tests that provide perfect correspondence with the current in vivo toxicity testing approaches}

The goal of toxicity testing, however it is pursued, is to insure safety of chemicals (or the presence of no more than some very small, societally tolerable level of risk) in exposed human populations. However, new methods and new paradigms are necessary to achieve this end. Tests systems need to be designed to answer questions regarding the pathways that are affected by chemicals; whether these pathways are as likely to be affected in humans as in the test system; the physiological consequences of alterations in these pathways; and the overall dose response curve for the effects. In this way in vitro test systems or in silico methods are not simply alternatives to animal tests, nor are they intended to be replacements for animal tests. Instead, they are intended to give specific answers to questions regarding hazards of chemical exposures expected in human populations. These new methods represent improvements on the present approaches, not some lesser position to be taken in the absence of available animal tests.

\section{In vitro methods cannot provide information regarding dose-response necessary for risk assessments}

The animal tests provide a dose response curve for the effects in these test animals. Only one point of this curve, i.e., the NOAEL/NOEL is used. Methods used for predicting lower dose responses, such as dividing by various uncertainty factors, are no more than conventions propped by tradition rather than informed predictions based on knowledge of the biology of the responses. How can these risk estimates be made more relevant for assessing expected consequences of exposures in humans? Another way to pose the question is: how will toxicity testing take into consideration the mode of action and relevant measures of tissue dose for risk extrapolations? We and others evaluating new toxicity testing strategies believe that the answer here is the development of high throughput systems that examine specific precursor responses, i.e., specific response biomarkers, in appropriate in vitro test systems.
Today, the field of contemporary toxicity testing needs well-validated suites of cellular systems that examine relevant biomarkers for critical physiological effects (Eisenbrand et al. 2002). Ideally, these biomarkers would enable the reliable estimation of precursor responses with known relevance for an adverse endpoint in humans. The consideration of these adverse endpoints will dictate the selection of appropriate in vitro cellular test systems. Such decisions can be facilitated by knowledge of the structural information on the presence of chemical functionalities known to be related to the endpoints of concern (i.e., QSAR methods).

The outcome of these carefully conducted in vitro tests would be the cellular response characteristics for the precursor response/biomarker over a wide concentration range. These in vitro approaches yield much denser dose-response relationships covering a broader range of concentrations than for those developed from animal testing. These studies would provide data-rich concentration- (or an internal dose-) response relationships. Finally, these relationships serve as the basis for calculating the external dose (or human exposure scenario) leading to the concentrations used in vitro. These in vitro to in vivo extrapolations can be done by the application of physiologically-based biokinetic modeling (Andersen 2003), a field that has been extensively explored by one of us (Blaauboer 2003). The result of such approaches will be a better estimation of the internal effective concentration at the target sites in humans than can be reached with studies in animals.

It is clear from this outline that the elements of this approach, i.e., the in vitro, in silico, and computational components, need to be placed in the framework of a comprehensive testing strategy. Hence, the notion that these elements will be replacing current animal models on a one-to-one basis is overly simplistic and impedes innovation.

\section{Approaches to introduce new methods for toxicity testing and risk assessment reflect consideration of cost reduction and decreased animal use rather than decisions taken on the basis of toxicological considerations}

On the contrary, the introduction of these new toxicity testing methodologies is intended to improve current risk assessment processes, making them more relevant by incorporating mechanistic information, accounting for differences in dose between in vitro and in vivo conditions, and focusing on human relevance (Goldberg and Hartung 2005). During the last decade a number of 
studies have been carried out, showing the characteristics of the above-described, strategic approaches (DeJongh et al. 1999; Gubbels-van Hal et al. 2005; Verwei et al. 2006). Although these studies had limitations and shortcomings, they showed that the concept is feasible. Estimates were made of the exposure scenarios that result in toxic reactions in vivo on the basis of information from in vitro toxicity testing, QSARs and kinetic modeling. It is also clear from these studies, that there should be more emphasis on human models rather than simply observing the exposures leading to adverse effects in animals. This redefinition of toxicity testing is absolutely necessary for improving human risk assessment. We look forward to replacing the socalled "gold standard" of animal toxicity testing by a strategy resulting in a more targeted risk analysis for human health endpoints. The time is ripe to move on to a platinum standard for toxicity testing.

\section{References}

Andersen ME (2003) Toxicokinetic modeling and its applications in chemical risk assessment. Toxicol Lett 138:9-27
Blaauboer BJ (2003) The integration of data on physico-chemical properties, in vitro-derived toxicity data and physiologically based kinetic and dynamic modelling as a tool in hazard and risk assessment. A commentary Toxicol Lett 138:161-171

Eisenbrand G, Pool-Zobel B, Baker V, Balls M, Blaauboer BJ, Boobis A, Carere A, Kevekordes S, Lhuguenot J-C, Pieters R, Kleiner J (2002) Methods of in vitro toxicology. Food Chem Toxicol 40:193-236

DeJongh J, Nordin-Andersson M, Ploeger B, Forsby A (1999) Estimation of systemic toxicity of acrylamide by integration of in vitro toxicity data with kinetic simulations. Toxicol Appl Pharmacol 158:261-268

Greim H, Arand M, Autrup H, Bolt HM, Bridges J, Dybing E, Glomot R, Foa V, Schulte-Hermann R (2006) Toxicological comments to the discussion about REACH. Arch Toxicol 80:121-124

Goldberg AM, Hartung T (2005) Protecting more than animals. Reducing animal suffering often has the unexpected benefit of yielding more rigorous safety tests. Scientific American January 2005, pp 84-91

Gubbels-van Hal WM, Blaauboer BJ, Barentsen HM, Hoitink MA, Meerts IA, van der Hoeven JC (2005) An alternative approach for the safety evaluation of new and existing chemicals, an exercise in integrated testing. Regul Toxicol Pharmacol 42:284-295

Verwei M, van Burgsteden JA, Krul CAM, van de Sandt JJM, Freidig AP (2006) Prediction of in vivo embryotoxic effect levels with a combination of in vitro studies and PBPK modelling. Toxicol Letters 165:79-87 